$\S=$

\title{
Correlation study of optical, mechanical and thermal properties of BAs material and its experimental energy gap
}

\author{
Salah Daoud * \\ Laboratory of Materials and Electronic Systems (LMSE), Faculty of Sciences and Technology, Mohamed Elbachir El Ibrahimi, Bordj \\ Bou Arreridj University, Bordj Bou Arreridj (34000), Algeria \\ *Corresponding author E-mail: salah_daoud07@yahoo.fr
}

\begin{abstract}
The object of this work is to study the correction between the optical, mechanical and thermal properties of boron arsenide (BAs) material and its experimental optical energy gap. The index of refraction, the high-frequency dielectric constant, the optical electronegativity, the bulk modulus, the micro-hardness, the plasmon energy, the Debye temperature, the melting temperature and the electronic polarizability of BAs were estimated from its energy gap. The results obtained are analyzed in comparison with available experimental and other theoretical data. My obtained results of the reflective index and the dielectric constant agree well with other theoretical data; whereas the bulk modulus, the microhardness, the Debye temperature, and the melting temperature are slightly lower than the experimental and other theoretical data. The electronic polarizability is slightly different than other theoretical ones from the literature.
\end{abstract}

Keywords: Wide-Gap Semiconductor Materials; Optical, Mechanical and Thermal Properties; Electronic Polarizability.

\section{Introduction}

Boron based materials $\mathrm{BX}(\mathrm{X}=\mathrm{P}, \mathrm{As})$ are two compounds, which belong to III-V group [1]. These later have attracted increased research attention over the past few years because of great technological interest for electronic and optical devices manufacturing $[1,2]$ and high temperature applications [1].

To best of our knowledge, BP and BAs semiconductors were experimentally obtained for the first time in 1958 by Perri et al. [3]. They found that both BP and BAs compounds crystallize in cubic zincblende structure, this structure consists of the interpenetrating FCC lattices, one displaced from other by a distance $(0.25,0.25$, $0.25)$ along the body diagonal. They found also that when it heated in an atmosphere of phosphorus, BP compound was stable to a temperature at least $1250{ }^{\circ} \mathrm{C}$; whereas BAs compound was stable to a temperature $920^{\circ} \mathrm{C}$.

Yadav et al. [2] used the plasma oscillation theory of solids formalism to study the high-frequency refractive index, optical susceptibility, electronic polarizability and crystal ionicity of some cubic zincblende and wurtzite type structured binary compounds. In order to study the dielectric constant and electronic polarizability of some binary and ternary tetrahedral materials, Verma et al. [4] proposed some efficacy models.

In our very recent work [5], a quasi-linear relationship was obtained between the high-frequency and static dielectric constants in some II-VI and III-V materials. Some other physical quantities such as: Knoop microhardness, homopolar and heteropolar energies, thermal conductivity, and melting point of boron based materials (BAs, and $\mathrm{BSb}$ ) are also the object of other our recent works [6- 8]. Using first principles calculations, the structural phase transition, elastic constants, sound velocity, and Debye temperature of BAs were studied under pressure up to $125 \mathrm{GPa}$ [9]. In this work, I would like to study the correlation between the experimental optical energy gap and the optical (index of refraction and high-frequency dielectric constant), the mechanical and thermal properties of BAs semiconductor. Firstly, the reflective index (n) was calculated from two empirical formulas, and then the corresponding high-frequency dielectric constant was determined. The total optical electronegativity difference, bulk modulus, micro hardness, plasmon energy, Debye temperature, melting temperature, and electronic polarizability were also predicted. The results obtained are compared with the experiment and other theoretical data from the literature.

\section{Theory, results and discussion}

\subsection{Optical properties}

The evaluation of refractive index of a semiconductor material is of considerable importance to the manufacturing of devices in optoelectronic applications. For several, elements, III-V, II-VI and I-VII types binary semiconductors with different structures; $\mathrm{Ku}$ mar and Singh [10] proposed a formula which relates a refractive index $(n)$ with the optical energy gap $\left(\mathrm{E}_{\mathrm{g}}\right)$, it is given as follows [10]:

$\mathrm{n}=\mathrm{K}\left(\mathrm{Eg}_{\mathrm{g}}\right)^{\mathrm{C}}$

Where $\mathrm{K}=3.3668$ and $\mathrm{C}=-0.32234$ are constants.

Anani et al. [11] reported that the refractive index (n) of a semiconductor is dependent on its optical energy gap $\left(\mathrm{E}_{\mathrm{g}}\right)$. They proposed a linear relationship between the index of refraction and the energy gap; it is given by the following expression [11]:

$$
n=(17-E g) / 5
$$


Replacing in Eq. (2) the experimental energy gap $\mathrm{E}_{\mathrm{g}}=1.46 \mathrm{eV}$ reported by Chu and Hyslop [12,13] of thin film BAs material, the predicted value of the refractive index was found to be 3.11 . It is very important to note that several authors [14-17] have quoted that BAs material has an indirect optical energy gap $\left(E_{g}=0.67\right.$ $\mathrm{eV}$ ), and they have mentioned also that this later value was obtained by Bross and Bader [18]. This is not true, because the authors of Ref. [18] have studied the ground state properties of diamond and cubic boron nitride materials, and not the properties of boron arsenide. The small indirect optical energy gap $\left(\mathrm{E}_{\mathrm{g}}=0.67\right.$ $\mathrm{eV}$ ) of BAs thin film material was also deduced by Chu and Hyslop [13].

In other very recent work [19], an important formula was established between the refractive index (n) and the optical electronegativity $\left(\Delta X^{*}\right)$, it was given as [19]

$$
\mathrm{n}=\mathrm{k}\left(\Delta \mathrm{X}^{*}+\mathrm{C}\right)^{\gamma}
$$

Where the parameters $\mathrm{k}, \mathrm{C}$ and $\gamma$ depend on the nature group of material. The relevant values of the constants $\mathrm{k}, \mathrm{C}$ and $\gamma$ for III-V semiconductors are $2.36,-0.13$ and -0.22 respectively [19].

The optical electronegativity indicates the nature of the bonding in the solids. Reddy et al. [20] studied the correlation between the optical electronegativity, the bulk modulus and the electronic polarizability in various binary systems and ternary chalcopyrite semiconductors. The optical electronegativity $\left(\Delta X^{*}\right)$ has been given as a function of the optical energy gap (Eg) as follows [20]:

$$
\Delta \mathrm{X}^{*}=(0.2688) \mathrm{Eg}
$$

Using the experimental optical energy gap $\mathrm{E}_{\mathrm{g}}=1.46 \mathrm{eV}$ reported by Chu and Hyslop [12], [13] of BAs material, the value of the optical electronegativity was found to be 0.4 . To the best of my knowledge, there is no data available in the literature on this quantity for this compound. So our findings on the optical electronegativity, perhaps is the first prediction and can be useful for future investigations of this compound. The value $(0.4)$ of the optical electronegativity of BAs material is equals exactly to that obtained for AlSb semiconductor [20].

Replacing in Eq. (4) the value $\left(\Delta X^{*}=0.4\right)$, the predicted value of $\mathrm{n}$ was found to be 3.15. It is in excellent accord with the value 3.11 obtained from Eq. (2). My calculated values of $n$ are listed in Table 1 and compared with other theoretical data [17], [21], [22]. As can be seen from Table 1 , my calculated values of $\mathrm{n}$ are in very good agreement with the theoretical ones (3.05) reported in Ref. [17] and (3.35) reported in Ref. [21] respectively, the deviation between our value (3.11) and that (3.05) of Ref. [17] is only around $1.96 \%$. At given optical frequency, the dielectric constant $\varepsilon$ and the refractive index $\mathrm{n}$ are related by Lorentz-Lorenz's formula, it is given by the following expression [8], [23]

$$
n^{2}=\varepsilon
$$

Using Eq. (5), our calculated values of $\varepsilon$ are 9.66 and 9.91 respectively. They are also listed in Table 1 and compared with other theoretical data [17], [21], [22], [24-26].

Table 1: Index of Refraction (n) and High-Frequency Dielectric Constant (ع) of BAs in Comparison with Available Data [17], [21], [22], [24-26] of the Literature. ${ }^{\mathrm{a}}$ Obtained from Eq. (2), ${ }^{\mathrm{B}}$ Obtained From Eq. (3).

\begin{tabular}{lll}
\hline Parameter & $\mathrm{n}$ & $\varepsilon$ \\
\hline This work & $3.11^{\mathrm{a}}{ }^{3} 3.15^{\mathrm{b}}$ & $9.66^{\mathrm{a}} 9.91^{\mathrm{b}}$ \\
Ref. [17] & 3.05 & 9.3 \\
Ref. [21] & 3.35 & 11.19 \\
Ref. [22] & 2.09 & - \\
Ref. [24] & - & 9.56 \\
Ref. [25] & - & 9.67 \\
Ref. [26] & - & 9.65 \\
\hline
\end{tabular}

As can be seen from Table 1, my calculated values (9.66 and 9.91) of $\varepsilon$ are also in very good agreement with other theoretical data. For example, the deviation between our obtained value (9.91) of $\varepsilon$ and the value (9.67) of the Ref. [25] is less than $2.5 \%$; whereas, the deviations between our obtained value (9.66) and the value (9.67) obtained by Labidi et al. [25] from first-principles fullpotential linearized augmented-plane wave calculations is less than $0.01 \%$. This very good agreement indicates the high correlation of the optical properties and energy gap in BAs material.

\subsection{Bulk modulus and microhardness}

In this part of the present work, and in order to estimate the bulk modulus $\mathrm{B}$ and the microhardness $\mathrm{H}$ of BAs compound at room temperature, I use some empirical formulas which have been proposed by Reddy et al. [27], and recently successfully applied to other boron based materials (BSb [8], and BBi [28]). In this model only one experimental data is required in the calculations it is the refractive index of the semiconductor. For some semiconductors (groups II-VI and III-V), the refractive index was related to the bulk modulus by the following relationship [8], [27], [28]

$\mathrm{B}(\mathrm{GPa})=\mathrm{K}_{1} \exp \left(\mathrm{K}_{2} \mathrm{n}\right)-\mathrm{K}_{3}$

Where $K_{1}, K_{2}$ and $K_{3}$ are constants. The relevant values of $K_{1}, K_{2}$ and $\mathrm{K}_{3}$ for III-V semiconductors are: $648.89,-0.3546$, and 130.33 , respectively [27]. Replacing my obtained value of the refractive index $(n=3.11)$ in Eq. (6), the value of the bulk modulus of BAs compound is given in Table 2 and compared with available theoretical data [29], [30], [31], and experimental result [32].

For the same semiconductors, the microhardness can be expressed as function the refractive index as follow [8], [27], [28]

$\mathrm{H}(\mathrm{GPa})=\mathrm{K}_{4} \exp \left(\mathrm{K}_{5} \mathrm{n}\right)-\mathrm{K}_{6}$

Where $\mathrm{K}_{4}, \mathrm{~K}_{5}$ and $\mathrm{K}_{6}$ are constants, the values of $\mathrm{K}_{4}, \mathrm{~K}_{5}$ and $\mathrm{K}_{6}$ for III-V materials are: $104.953,-0.3546$ and 26.82 , respectively [27]. Replacing the numerical values of $\mathrm{K}_{4}, \mathrm{~K}_{5}$ and $\mathrm{K}_{6}$ and the value $(n=3.11)$ in Eq. (7), the obtained value of the microhardness of BAs compound are given in Table 2 and compared with other theoretical data [7], [29]. It can be seen, that my calculated values of bulk modulus, and microhardness of BAs semiconductor are slightly lower than other theoretical calculations [7], [29], [30], [31] and experimental result [32].

Table 2: Bulk Modulus and Microhardness of BAs Semiconductor, in Comparison with Available Data of the Literature [7], [29-32]. ${ }^{\text {a }}$ Obtained from Eq. (7), ${ }^{\mathrm{b}}$ Obtained from Eq. (9)

\begin{tabular}{lll}
\hline Parameter & $\mathrm{B}(\mathrm{GPa})$ & $\mathrm{H}(\mathrm{GPa})$ \\
\hline This work & 110.44 & $8.02^{\mathrm{a}} 7.11^{\mathrm{b}}$ \\
Ref. [7] & - & 17.94 and 23.82 \\
Ref. [29] & From 149.53 to 192.91 & From 12.1 to 17.23 \\
Ref. [30] & 137.61 & 16.5 \\
Ref. [31] & 138 & - \\
Ref. [32] Exp & $148 \pm 6$ & - \\
\hline
\end{tabular}

To study the correlation between the bulk modulus B and the energy gap $E_{g}$ Reddy et al. [23] proposed linear relationships. For several III-V, II-IV-V $\mathrm{V}_{2}$ and I-III-VI 2 group semiconductors, an important formula relates the bulk modulus B and the energy gap $\mathrm{E}_{\mathrm{g}}$ was established, it is given as follow [23]:

$\mathrm{B}(\mathrm{GPa})=\mathrm{C}_{1} \mathrm{Eg}+\mathrm{C}_{2}$

The relevant values of $\mathrm{C}_{1}$ and $\mathrm{C}_{2}$ for III-V, II-IV-V $\mathrm{V}_{2}$ and I-III-VI group semiconductors are: 13.89 and 46.90 respectively [23].

Using the experimental energy gap $E_{\mathrm{g}}=1.46 \mathrm{eV}$ reported by Chu and Hyslop [12], [13] of BAs material, the value of the bulk modulus B is found to be $67.18 \mathrm{GPa}$. Unfortunately, this result do not agree well with my previous result (110.44 GPa) obtained from Eq (6), and the experimental one (148 $\pm 6 \mathrm{GPa})$ reported in Ref. [32]. 
For some elemental of group IV, II-VI, III-V, II-IV-V 2 and I-III$\mathrm{VI}_{2}$ group materials, an important formula relates the microhardness $H$ and the plasmon energy $\hbar \omega_{p}$ was established by Kumar et al [30], it is given by the following expression [30]

$\mathrm{H}=\mathrm{K}_{9}\left(\hbar \omega_{\mathrm{p}}\right)^{2.3333}-\mathrm{K}_{10}$

For III-V compounds, the values of the constants $\mathrm{K}_{9}$ and $\mathrm{K}_{10}$ are 0.0202 and 5.743 respectively.

Replacing my obtained value of the plasmon energy $\hbar \omega_{\mathrm{p}}=15.91$ $\mathrm{eV}$ (Please see Section 2.3) in the formula of Eq. (9), the value of $\mathrm{H}$ of BAs compound is found to be $7.11 \mathrm{GPa}$. It is also given in Table 2 and compared with other theoretical data [7], [29], and [30]. It can be seen, that my calculated value of the microhardness obtained from Eq. (9) is in agreement with my previous result (8.02 GPa) obtained from Eq. (7); but, it is also slightly lower than other theoretical ones [7], [29], and [30].

\subsection{Debye temperature and melting temperature}

The Debye temperature $\left(\theta_{\mathrm{D}}\right)$ and the melting temperature $\left(\mathrm{T}_{\mathrm{m}}\right)$ are two fundamental thermophysical properties of materials. The Debye temperature is usually used to distinguish between low and high temperature regions [33].

In our recent work [28], the plasmon energy $\left(\hbar \omega_{p}\right)$ used with success to predict the Debye temperature $\left(\theta_{\mathrm{D}}\right)$ of $\mathrm{BBi}$ compound. Usually, the plasmon energy was given as function of the effective number of valence electrons taking part in plasma oscillations $\mathrm{Z}$, the specific gravity $\sigma$ and the molecular weight $\mathrm{W}$ by the following relationship [30]:

$$
\hbar \omega_{\mathrm{p}}(\mathrm{eV})=28.8(\mathrm{Z} \sigma / \mathrm{W})
$$

The plasmon energy was also related to the refractive index (n) by the following relationship [8], [27], [28]

$$
\hbar \omega_{\mathrm{p}}(\mathrm{eV})=\mathrm{K}_{7} \exp \left(\mathrm{K}_{8} \mathrm{n}\right)
$$

Where $\mathrm{K}_{7}$ and $\mathrm{K}_{8}$ are constants. The numerical values of the constants $\mathrm{K}_{7}$ and $\mathrm{K}_{8}$ for III-V group are: 47.924 , and -0.3546 , respectively [27]. Replacing the value $(\mathrm{n}=3.11)$ in Eq. (11), the plasmon energy of BAs was found to be $15.91 \mathrm{eV}$; it is relatively lower than the theoretical calculations (17.77 to $20.11 \mathrm{eV}$ ) reported in Ref. [29], and (20.12 eV) obtained by Kumar et al. [30].

The Debye temperature $\left(\theta_{\mathrm{D}}\right)$ and the plasmon energy $\left(\hbar \omega_{\mathrm{p}}\right)$ are related by [28]:

$\theta_{\mathrm{D}}=-\mathrm{K}_{9}+\mathrm{K}_{10}\left(\hbar \omega_{\mathrm{p}}\right)$

The values of the constants $\mathrm{K}_{9}$ and $\mathrm{K}_{10}$ are respectively: 800.88 and 77.48 for III-V group [28]. Using Eq. (12), the value of $\theta_{D}$ was calculated, it is around $432 \mathrm{~K}$. The melting temperature $\left(\mathrm{T}_{\mathrm{m}}\right)$ was proposed, it is given by the following formula [9], [28]:

$\mathrm{T}_{\mathrm{m}}=\left(\theta_{\mathrm{D}}+\mathrm{K}_{12}\right) / \mathrm{K}_{13}$

The values of $K_{12}$ and $K_{13}$ are respectively: 153.40 and 0.354 for III-V group. The obtained value of $\mathrm{T}_{\mathrm{m}}$ was calculated, it is around 1653.2 K. These two values are slightly lower than the results of our previous works [6], [9] (Table 3) and references therein.

\subsection{Electronic polarizability}

If we know the refractive index $\mathrm{n}$ and the crystal density $\mathrm{g}$ of any crystalline material, it's possible to predict the electronic polarizability $\alpha_{p}$ by using the following formula [34]

$$
\alpha_{p}=\frac{\left(n^{2}-1\right) \cdot M \cdot 10^{-24}}{\left(n^{2}+2\right) \cdot g \cdot(2 \cdot 53)}
$$

Where, $\mathrm{n}$ is refractive index, $\mathrm{M}$ is the molecular weight and $\mathrm{g}$ is the density.

Using the X-ray crystal density $\left(\mathrm{g}=5.224 \mathrm{~g} / \mathrm{cm}^{3}\right)$ reported by Adachi [35], and my value $(n=3.11)$, the value of $\alpha_{p}$ of BAs compound was found to be: $4.82 \AA^{3}$, it is slightly larger than the value (4.41 $\AA^{3}$ ) reported in Ref. [30]. Another formula was given by Reddy et al. [23], which relates the electronic polarizability $\alpha_{p}$ and the bulk modulus B of several materials, it was given as [23]:

$$
\alpha_{p}=(0.395) \times 10^{-24} \frac{\left[(5.563-0.033 B)^{2}-1\right] \cdot M}{\left[(5.563-0.033 B)^{2}+2\right] \cdot g}
$$

Using the density $\left(\mathrm{g}=5.224 \mathrm{~g} / \mathrm{cm}^{3}\right)$ reported by Adachi. [35], and my obtained value of the bulk modulus $\mathrm{B}=110.44 \mathrm{GPa}$, the value of the electronic polarizability $\alpha_{p}$ is found to be $3.06 \AA^{3}$. Our calculated value of $\alpha_{\mathrm{p}}$ is listed also in Table 3. As can be seen from Table 3, my obtained value ( $3.06 \AA^{3}$ ) of $\alpha_{p}$ of BAs compound is slightly lower than the value $4.08 \AA^{3}$ of Verma et al. [4], the values $4.05-4.51 \AA^{3}$ reported by Shaileshkumar [29], and the value $4.41 \AA^{3}$ reported in Ref. [30].

\begin{tabular}{|c|c|c|c|}
\hline Parameter & $\theta_{\mathrm{D}}(\mathrm{K})$ & $\mathrm{T}_{\mathrm{m}}(\mathrm{K})$ & $\alpha_{p}\left(\AA^{3}\right)$ \\
\hline This work & 432 & 1653.2 & $4.82^{\mathrm{a}} 3.06^{\mathrm{b}}$ \\
\hline Ref. [4] & - & - & 4.08 \\
\hline Ref. [6] & 657.37 & 2264.3 & - \\
\hline Ref. [9] & 698.16 & 2405.53 & - \\
\hline Ref. [29] & - & - & From 4.05 to 4.51 \\
\hline Ref. [30] & - & - & 4.41 \\
\hline
\end{tabular}

Table 3: Debye Temperature $\left(\Theta_{d}\right)$, Melting Temperature $\left(T_{m}\right)$, and Electronic Polarizability $\alpha_{p}$ of Bas, Compared to Other Data [4], [6], [9], [29] And [30]. ${ }^{a}$ Obtained from Eq. (14), ${ }^{\mathrm{b}}$ Obtained from Eq. (15)

It is important to note that the value $\left(\alpha_{p}=4.08 \AA^{3}\right)$ obtained by Verma et al. [4] is not precise, because the authors of Ref. [4] used the value $(\mathrm{d}=2.04 \AA)$ as nearest neighbor distance for BAs. The exact value of the nearest neighbor distance of BAs semiconductor can be deduced from the experimental lattice parameter $(\mathrm{a}=4.777$ $\AA$ ) [3], [35]. The correct value of the nearest neighbor distance for boron arsenide semiconductor becomes $(\mathrm{d}=2.07 \AA)$ [31], which gives a value $\alpha_{p}=4.25 \AA^{3}$. This later is in general slightly higher than the value $\left(\alpha_{p}=4.08 \AA^{3}\right)$ quoted by Verma et al. [4].

\section{Summary and conclusions}

In the present paper, I studied the correction between the optical and mechanical properties of BAs material and its experimental optical energy gap. Firstly, I have determined the reflective index and the corresponding high-frequency dielectric constant, the results obtained agree well with other theoretical data from the literature. The optical electronegativity was also calculated. The value was found to be: 0.4 . It equals exactly to that of AlSb compound [20]. To best of my knowledge, there is no data available through the literature on this quantity for this compound. Hence, my result is prediction and may serve as a reference, and still await other theoretical confirmations.

In addition, the bulk modulus, the micro hardness, the plasmon energy, the Debye temperature, and the melting temperature are also obtained. Despite my obtained values of the reflective index and the high-frequency dielectric constant agree well with other theoretical data from the literature, in general, my results of B, H, $\hbar \omega_{\mathrm{p}}, \theta_{\mathrm{D}}$ and $\mathrm{T}_{\mathrm{m}}$ are slightly lower than the experimental and other theoretical data from the literature. Ultimately, the electronic polarizability of BAs was also obtained from two formulas; the results obtained are slightly different than other theoretical ones.

Despite the optical electronegativity of BAs is equals exactly to that obtained for AlSb material, it is very important to note that the electronic polarizability $\left(4.82 \AA^{3}\right.$ and $\left.3.06 \AA^{3}\right)$ of BAs is very lower than that $\left(10.53 \AA^{3}\right)$ [20] of AlSb material. 


\section{References}

[1] M. Sarwan, P. Bhardwaj, and S. Singh, "Zinc-blende to rock-salt structural phase transition of BP and BAs under high pressure", Chemical Physics, Vol. 426, (2013), pp. 1-8. https://doi.org/10.1016/j.chemphys.2013.09.008.

[2] D. S. Yadav, C. Kumar, J. Sigh, Parashuram, and G. Kumar, "Optoelectronic properties of zinc blende and wurtzite structured binary solids", Journal of Engineering and Computer Innovations, Vol.3, No. 2, (2012), pp. 26-35.

[3] A. J. Perri, S. LaPlaca, and B. Post, "New Group III-Group V Compounds: BP and BAs", Acta Crystallographica, Vol. 11, (1958), pp. 310-310. https://doi.org/10.1107/S0365110X58000827.

[4] A. S. Verma, R. K. Singh, and S. K. Rathi, "An empirical model for dielectric constant and electronic polarizability of binary (ANB8$\mathrm{N}$ ) and ternary (ANB2+ NC2 7- N) tetrahedral semiconductors", Journal of Alloys and Compounds, Vol. 486, No. 1-2, (2009), pp. 795-800. https://doi.org/10.1016/j.jallcom.2009.07.067.

[5] S. Daoud, A. Bencheikh, and L. Belagraa, "Quasi-linear correlation between high-frequency and static dielectric constants in II-VI and III-V semiconductors ", International Journal of Physical Research, Vol. 5, No.1, (2017), pp. 4-6.

[6] S. Daoud, "Sound velocities and thermal properties of BX (X=As, $\mathrm{Sb})$ compounds", International journal of scientific world, Vol.3, No.1, (2015), pp. 43-48. https://doi.org/10.14419/ijsw.v3i1.4039.

[7] S. Daoud, "Empirical study of elastic properties of $\mathrm{BX}(\mathrm{X}=\mathrm{As}, \mathrm{Sb})$ materials", International journal of scientific world, Vol.3, No.1, (2015), pp. 37-42. https://doi.org/10.14419/ijsw.v3i1.4022.

[8] S. Daoud and A. Latreche, "Optical properties and electronic polarizability of boron-antimonide semiconductor", International Journal of Physical Research, Vol. 5, No.2, (2017), pp. 43-45.

[9] S. Daoud, A. Bioud, and N. Bouarissa, "Structural phase transition, elastic and thermal properties of boron arsenide: Pressure-induced effects", Materials Science in Semiconductor Processing, Vol. 31, (2015), pp. 124-130. https://doi.org/10.1016/j.mssp.2014.11.024.

[10] V. Kumar and J. K. Singh, "Model for calculating the refractive index of different materials", Indian Journal of Pure and Applied Physics, Vol.48, (2010), pp. 571-574. http://www.nusod.org/piprek/ guden $96 \mathrm{msmse}$.pdf.

[11] M. Anani, C. Mathieu, S. Lebid, Y. Amar, Z. Chama, and H. Abid, "Model for calculating the refractive index of a III-V semiconductor", Computational Materials Science, Vol.41, No.4, (2008), pp. 570-575. https://doi.org/10.1016/j.commatsci.2007.05.023.

[12] T. L. Chu and A. E. Hyslop, "Crystal growth and properties of boron monoarsenide", Journal of Applied Physics, Vol.43, No.2, (1972) 276- 279. https://doi.org/10.1063/1.1661106.

[13] T. L. Chu and A. E. Hyslop, "Preparation and properties of boron arsenide films", Journal of The Electrochemical Society, Vol. 121, No.3, (1974), pp. 412-415. http://jes.ecsdl.org/content/121/3/412. full.pdf. https://doi.org/10.1149/1.2401826.

[14] A. Zaoui and F. El Haj Hassan, "Full potential linearized augmented plane wave calculations of structural and electronic properties of BN, BP, BAs and BSb", Journal of Physics: Condensed Matter, Vol. 13, No.2, (2001), pp. 253-262. https://doi.org/10.1088/0953$8984 / 13 / 2 / 303$

[15] H. Meradji, S. Labidi, S. Ghemid, S. Drablia, and B. Bouhafs, " First principles calculations of structural, electronic and optical properties of BAs1-xPx alloy", Physics Procedia , Vol. 2, (2009), pp. 933-940. https://doi.org/10.1016/j.phpro.2009.11.046.

[16] L. Hamioud, A. Boumaza, S. Touam, H. Meradji, S. Ghemid, F. E Haj Hassan, R. Khenata, and S. Bin Omran, " First-principles calculations of the structural, electronic, optical and thermal properties of the BNxAs1-x alloys ", Philosophical Magazine, Vol. 96, No. 16, (2016), pp. 1694-1711.

[17] E. Viswanathan, M. Sundareswari, D. S. Jayalakshmi, M. Manjula and S. Krishnaveni, "Structural, electronic, mechanical, thermal and optical properties of $\mathrm{B}(\mathrm{P}, \mathrm{As}) 1-\mathrm{xNx} ;(\mathrm{x}=0,0.25,0.5,0.75,1)$ alloys and hardness of $\mathrm{B}(\mathrm{P}, \mathrm{As})$ under compression using DFT calculations", Indian journal of physics, Vol. 91, No.9, (2017), pp. 999 1011. https://link.springer.com/article/10.1007/s12648-017-0996-0. https://doi.org/10.1007/s12648-017-0996-0.

[18] H. Bross, R. Bader, "Calculation of the Ground State Properties of Diamond and Cubic Boron Nitride", Physica Status Solidi B, Vol.191, No. 2, (1995), pp. 369-385. https://doi.org/10.1002/pssb.2221910212.

[19] A. Latreche and S. Daoud, "Modified expression for calculating refractive index of ANB8-N type binary semiconductors ", International Journal of Physical Research, Vol.4, No.2, (2016), pp. 48-51.
[20] R.R. Reddy, Y.N. Ahammed, K.R. Gopal, P.A. Azeem, T.V.R. Rao, and P.M. Reddy, " Optical electronegativity, bulk modulus and electronic polarizability of materials", Optical Materials, Vol. 14, No. 4, (2000), pp. 355-358.

[21] A. Zaoui, S. Kacimi, A. Yakoubi, B. Abbar, and B. Bouhafs, "Optical properties of BP, BAs and BSb compounds under hydrostatic pressure", Physica B, Vol. 367, No. 1-4, (2005), pp. 195-204 https://doi.org/10.1016/j.physb.2005.06.018.

[22] A. S. Verma, "Correlation between ionic charge and the optical properties of zinc blende and complex crystal structured solids", Physica Status Solidi B, Vol.246, No. 1, (2009), pp. 192-199. https://doi.org/10.1002/pssb.200844242.

[23] R.R. Reddy, K. Rama Gopal, K. Narasimhulu, L. Siva Sankara Reddy, K. Raghavendra Kumar, G. Balakrishnaiah, and M. Ravi Kumar, "Interrelationship between structural, optical, electronic and elastic properties of materials", Journal of Alloys and Compounds, Vol. 473, (2009), pp. 28-35.

[24] K. Bouamama, P. Djemia, N. Lebgaa and K. Kassali, "Ab initio calculation of the lattice dynamics of the Boron group-V compounds under high pressure", High Pressure Research, Vol. 27, No. 2 (2007), pp. $269-277$

[25] S. Labidi, H. Meradji, S. Ghemid, S. Meçabih, B. Abbar, " Pressure dependence of electronic and optical properties of zincblende BP, BAs and BSb compounds", Journal of Optoelectronics and Advanced Materials, Vol. 11, No. 7, (2009), pp. 994 - 1001. http://joam.inoe.ro/index.php?option=magazine $\&$ op=view $\& i d u=19$ 89 \&catid $=40$.

[26] D. Touat, M. Ferhat and A. Zaoui, "Dynamical behavior in the boron III-V group: a first-principles study", Journal of Physics: Condensed Matter, Vol. 18, No. 15, (2006), pp. 3647-3654. https://doi.org/10.1088/0953-8984/18/15/011.

[27] R. Reddy, Y. Nazeer Ahammed, P. Abdul Azeem, K. Rama Gopal, B. Sasikala Devi, and T. V. R. Rao, "Dependence of physical parameters of compound semiconductors on refractive index", Defense Science Journal, Vol. 53, No. 3, (2003), pp. 239-248 http://www.publications.drdo.gov.in/ojs/index.php/dsj/article/view/ 2272. https://doi.org/10.14429/dsj.53.2272.

[28] S. Daoud, N. Bioud, N. Lebga, and R. Mezouar, "Optoelectronic and thermal properties of boron- bismuth compound", International Journal of Physical Research, Vol.2, No.2, (2014), pp. 27-31. https://doi.org/10.14419/ijpr.v2i2.2760

[29] V. P. Shaileshkumar, "Application of pseudopotential theory to certain binary, ternary and quaternary semiconductors", Thesis of Doctor of Philosophy in Physics, Sardar Patel University, India (January- 2012). http://hdl.handle.net/10603/7350.

[30] V. Kumar, A.K. Shrivastava, and Vijeta Jha, "Bulk modulus and microhardness of tetrahedral semiconductors", Journal of Physics and Chemistry of Solids, Vol. 71, (2010), pp. 1513-1520. https://doi.org/10.1016/j.jpcs.2010.07.012.

[31] M. Kitamura, S. Muramatsu and W. A. Harrison, "Elastic properties of semiconductors studied by extended Hückel theory", Physical Review B, Vol. 46, No. 3, (1992), pp. 1351-1357. https://doi.org/10.1103/PhysRevB.46.1351.

[32] R.G. Greene, H. Luo, A.L. Ruoff, S.S. Trail, and F.J. DiSalvo, " Pressure induced metastable amorphisation of BAs: evidence for a kinetically frustrated phase transformation", Physical Review Letters, Vol. 73, (1994), pp. 2476-2479.

[33] S. Daoud, "Thermal properties of cubic zincblende thallium phosphide from quasi-harmonic Debye model approximation", International Journal of Physical Research, Vol. 5, No.1, (2017), pp. 14-16.

[34] A. S. Verma, "Electronic and optical properties of rare-earth chalcogenides and pnictides", African Physical Review, Vol.3, (2009), pp. 11-20. www.aphysrev.org/index.php/aphysrev/article/ download $/ . . . / 151$.

[35] S. Adachi, "Properties of Group-IV, III-V and II-VI Semiconductors", John Wiley \& Sons, England, (2005). ISBN 0-470-09032-4 https://doi.org/10.1002/0470090340. 